\title{
Adenosina trifosfato bioluminescência para avaliação da limpeza de superfícies: uma revisão integrativa
}

\author{
Adenosine triphosphate bioluminescence to evaluate the effectiveness \\ of surface cleaning: an integrative review \\ Bioluminiscencia adenosina trifosfato para evaluar la eficacia de la limpieza \\ de superficies: una revisión integrativa
}

\author{
Adriana Cristina de Oliveira', Roberta El Hariri Viana" \\ ' Universidade Federal de Minas Gerais, Escola de Enfermagem, \\ Departamento de Enfermagem Básica. Belo Horizonte-MG, Brasil. \\ "Universidade Federal de Minas Gerais, Escola de Enfermagem, \\ Programa de Pós-Graduação em Enfermagem. Belo Horizonte-MG, Brasil.
}

Submissão: 06-05-2013 Aprovação: 01-11-2014

\section{RESUMO}

Objetivo: Identificar na literatura indicações e controvérsias do ATP bioluminescência para avaliação da efetividade da limpeza de superfícies em estabelecimentos de saúde. Método: Revisão integrativa da literatura, entre 2000 e 2012, nas bases de dados MEDLINE, LILACS, Science Direct, SCOPUS e Isi Web of Knowledge. Resultados: Selecionou-se para esta revisão 15 artigos. O ATP bioluminescência foi apontado como importante recurso educacional e método complementar à inspeção visual e às análises microbiológicas na avaliação da efetividade da limpeza. A impossibilidade de indicar a contaminação da superfície por micro-organismos viáveis, a interferência por substâncias químicas e a dificuldade de interpretação dos resultados constituem as principais controvérsias para o uso deste nos serviços de saúde. Conclusão: Apesar de constituir importante recurso na avaliação da limpeza de superfícies, mais estudos são necessários para incorporação efetiva do método nos serviços de saúde. Descritores: Trifosfato de Adenosina; Serviço Hospitalar de Limpeza; Contaminação.

\begin{abstract}
Objective: To identify indications and controversies in the literature of the use of ATP bioluminescence to evaluate the effectiveness of surface cleaning in healthcare facilities. Method: Integrative literature review between 2000 and 2012 in the following databases: MEDLINE, LILACS, Science Direct, SCOPUS and Isi Web of Knowledge. Results: were selected for this review 15 articles. The ATP bioluminescence was considered an important educational resource and complementary method to visual inspection and microbiological evaluation of the effectiveness of cleaning. The impossibility to indicate surface contamination by microorganisms, interference by chemicals and the difficulty of interpreting the results constitute the main controversies in the use of ATP in health services. Conclusion: Although this is an important resource in the evaluation of surface cleaning, more studies are necessary for effective incorporation of the method in health services.
\end{abstract}

Key words: Adenosine Triphosphate; Hospital Housekeeping; Contamination.

\section{RESUMEN}

Objetivo: Identificar en la literatura las indicaciones y controversias sobre el uso de la bioluminiscencia ATP para evaluar la eficacia de la limpieza de superficies en los servicios de salud. Método: Revisión integrativa de la literatura, entre 2000 y 2012, en las siguientes bases de datos: MEDLINE, LILACS, Science Direct, SCOPUS e ISI Web of Knowledge. Resultados: Se seleccionaron para esta revisión 15 artículos. La bioluminiscencia del ATP se considera un importante recurso educativo y método complementario a la inspección visual y la análisis microbiológica de la evaluación de la efectividad de la limpieza. La imposibilidad de indicar contaminación de la superficie por los microorganismos, la interferencia por los productos químicos y la dificultad de interpretar los resultados constituyen las principales controversias en la utilización de ATP en los servicios de salud. Conclusión: Aunque esto es un elemento importante en la evaluación de limpieza de superficies, se necesitan más estudios para incorporación eficaz del método en los servicios de salud.

Palabras clave: Adenosina Trifosfato; Servicio de Limpieza em Hospital; Contaminación. 


\section{INTRODUÇÃO}

As infecções relacionadas a assistência a saúde (IRAS) representam um risco substancial à segurança do paciente no processo assistencial, sendo frequentemente relacionada à contaminação cruzada. Apesar de não estar claro o papel do ambiente na aquisição de potenciais patógenos, diversos micro-organismos de relevância epidemiológica têm sido isolados de diferentes locais no ambiente hospitalar. Acredita-se que uma vez contaminadas, estas superfícies podem favorecer a disseminação de bactérias ${ }^{(1-7)}$.

As medidas de higiene ambiental em estabelecimentos de saúde são consideradas parte primordial das múltiplas estratégias necessárias para a prevenção e o controle das IRAS. Contudo, evidências apontam que o processo de limpeza nas instituições de saúde tem sido ineficaz, uma vez que não atende aos propósitos ao qual se destina, como a redução dos micro-organismos presentes nas superfícies ${ }^{(8-13)}$.

Acrescenta-se ao exposto a importância dos métodos para avaliar a efetividade da limpeza ambiental, sendo o mais conhecido a inspeção visual, como também mais utilizado, além da aplicação de tintura fluorescente, adenosina trifosfato (ATP) bioluminescência e análises microbiológicas, tais como a contagem de colônias aeróbicas totais (ACC) e a identificação de organismo indicador ${ }^{(10,14-5)}$.

Dentre estes, o ATP bioluminescência tem se configurado como método amplamente utilizado na indústria alimentícia para validar e monitorizar a limpeza ambiental. Todavia, sua utilização nos estabelecimentos de saúde é, ainda, incipiente ${ }^{(11,16)}$.

De forma semelhante à área alimentícia, para avaliar a limpeza ambiental em estabelecimentos de saúde o método consiste da reação entre a enzima luciferase e as moléculas de ATP, derivadas de matéria orgânica microbiana e não microbiana, recuperadas da superfície a partir de swabs. As concentrações de ATP são quantificadas por um luminômetro e os resultados são expressos em unidade relativa de luz (URL) do inglês relative light units. Valores de referência de 25 a 500 URL tem sido propostos para avaliar a eficácia do processo de limpeza ${ }^{(7,17)}$.

Comparado às análises microbiológicas tradicionais, este oferece como vantagem resultados rápidos, obtidos em até dois minutos, associados à técnica simples e de fácil emprego. Entretanto, controvérsias relacionadas à sensibilidade e especificidade do método e à interpretação dos seus resultados, têm se constituído em importantes empecilhos para a incorporação efetiva do sistema de ATP nos serviços de saúde(7,11-2,18-22).

Diante do interesse na adoção de métodos efetivos para avaliar a limpeza de superfícies, especialmente aqueles quantitativos, e da recente introdução do ATP bioluminescência nos serviços de saúde, tornou-se relevante realizar a presente revisão integrativa com o objetivo de identificar na literatura as indicações e controvérsias do método de ATP bioluminescência para avaliação da efetividade da limpeza de superfícies em estabelecimentos de saúde.

\section{MÉTODO}

Trata-se de um estudo de revisão integrativa da literatura, que tem por finalidade reunir e sintetizar resultados de pesquisas sobre um determinado tema ou questão, de maneira sistemática e ordenada, contribuindo para o aprofundamento deste e para a prática baseada em evidência ${ }^{(23)}$.

A construção da revisão integrativa contemplou as seguintes etapas: identificação do tema e definição da questão

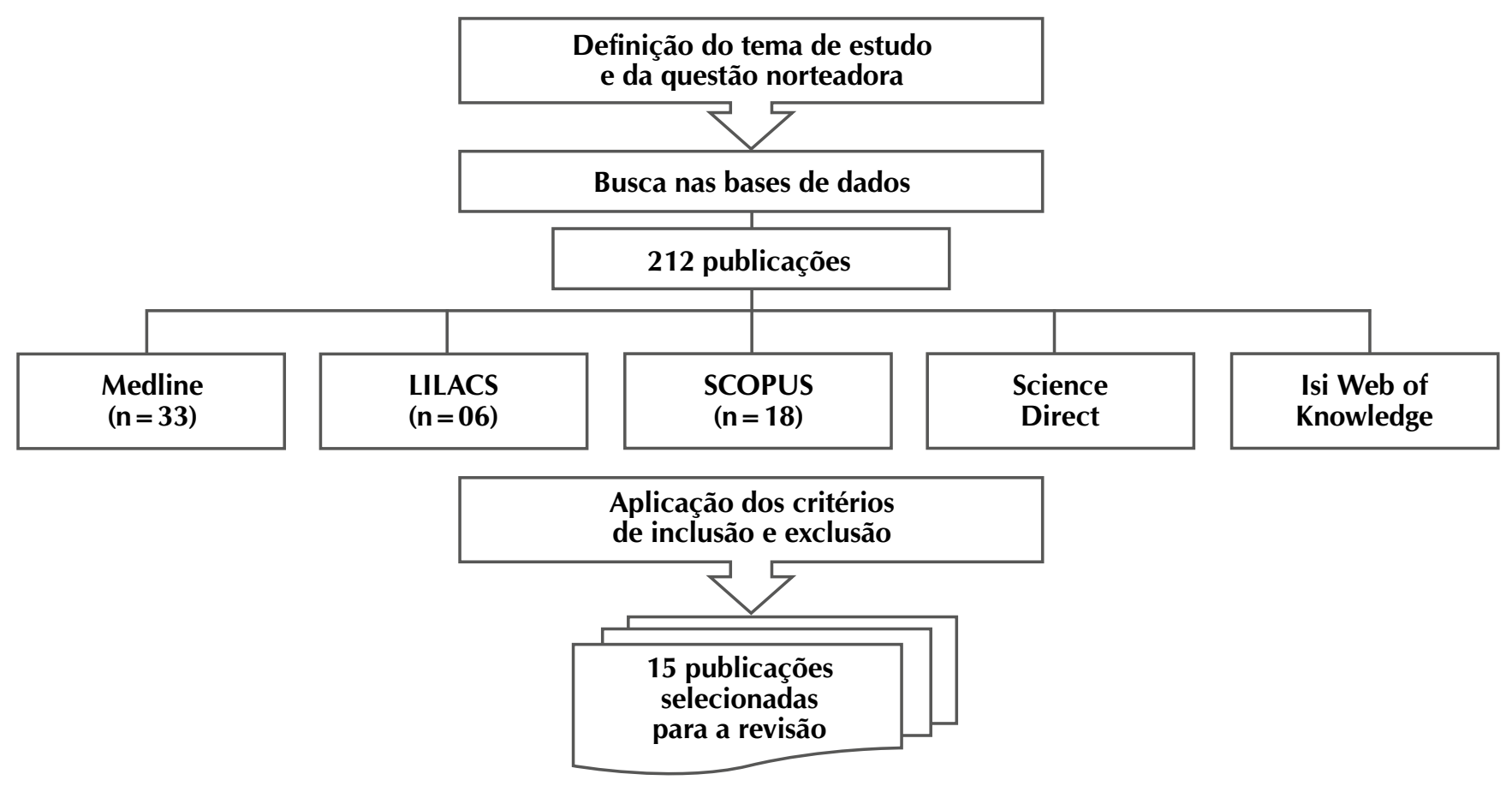

Figura 1 - Fluxograma representativo da seleção dos artigos incluídos na revisão integrativa 
de pesquisa, estabelecimento de critérios para inclusão e exclusão dos estudos (amostragem), definição das informações a serem extraídas dos artigos selecionados, avaliação dos estudos incluídos na revisão, interpretação dos resultados e apresentação da revisão.

Para nortear o presente estudo formulou-se a seguinte questão: Quais as indicações e controvérsias do uso do ATP bioluminescência para a avaliação da efetividade da limpeza de superfícies em estabelecimentos de saúde?

Para a seleção dos artigos foram utilizadas as seguintes bases de dados, a saber: Bibliografia Médica (MEDLINE), Literatura Latino Americana e do Caribe em Ciências da Saúde (LILACS), Science Direct, SCOPUS (Database of research literature) e plataforma virtual de pesquisa Isi Web of Knowledge. Utilizaram-se os descritores disponíveis no Decs/Mesh: adenosine triphosphate, trifosfato de adenosina, hospital housekeeping, serviço hospitalar de limpeza, contamination, contaminação.

Os critérios de inclusão das publicações selecionadas para a presente revisão foram: artigos originais publicados em inglês ou português, com referência ao uso do ATP bioluminescência em superfícies de estabelecimentos de saúde, no período compreendido entre 2000 e 2012. Optou-se por trabalhos publicados neste período por se tratar de tema com abordagem ainda escassa.

A busca foi realizada pelo acesso on-line, entre janeiro e março de 2013, e, inicialmente, foram obtidos 212 artigos.
Após a leitura criteriosa do título e resumo desses, foram excluídos aqueles que não se relacionavam ao tema (170), bem como os repetidos nas bases de dados e os artigos não originais (27). Utilizando os critérios de inclusão, a amostra final desta revisão foi constituída de 15 artigos, conforme evidenciado na Figura 1.

Para a coleta dos dados dos artigos que foram incluídos nesta revisão, foi elaborado um formulário contemplando os seguintes itens: identificação do artigo original, objetivos, características metodológicas do estudo, principais resultados e conclusões.

Para análise e posterior síntese das publicações foi utilizado um quadro sinóptico construído para esse fim, o qual contemplou os seguintes aspectos: nome do artigo, material e método, resultados e recomendações/conclusões.

A apresentação da revisão e a discussão dos dados foram realizadas de forma descritiva a fim de permitir ao leitor a avaliação crítica dos resultados obtidos e a sua aplicabilidade nas práticas de saúde.

\section{RESULTADOS}

Na análise dos 15 artigos incluídos na presente revisão, observou-se um predomínio de publicações provenientes do Reino Unido 46,6\% (7/15) e Estados Unidos 40\% (6/15), seguidos da Irlanda 6,7\% (1/15) e Brasil 6,7\% (1/15). O Quadro 1 apresenta as especificações de cada um dos artigos.

Quadro 1 - Publicações incluídas na revisão integrativa segundo o título do artigo, país, autores e periódico

\begin{tabular}{|c|c|c|c|}
\hline Título do artigo & País & Autores & $\begin{array}{c}\text { Periódicos } \\
\text { (volume, número, página, ano) }\end{array}$ \\
\hline $\begin{array}{l}\text { Monitoring the effectiveness of } \\
\text { hospital cleaning practices by } \\
\text { use of an adenosine triphosphate } \\
\text { bioluminescence assay. }\end{array}$ & Estados Unidos & Boyce JM. & $\begin{array}{l}\text { Infect Control Hosp } \\
\text { Epidemiol.30:678-84, } 2009 .\end{array}$ \\
\hline $\begin{array}{l}\text { An evaluation of hospital cleaning } \\
\text { regimes and standards. }\end{array}$ & Reino Unido & $\begin{array}{l}\text { Griffith CJ, Cooper RA, } \\
\text { Gilmore J, Davies C, Lewis M. }\end{array}$ & J Hosp Infect.45:19-28, 2000. \\
\hline $\begin{array}{l}\text { Who is really caring for your } \\
\text { environment of care? Developing } \\
\text { standardized cleaning procedures } \\
\text { and effective monitoring } \\
\text { techniques. }\end{array}$ & Estados Unidos & $\begin{array}{l}\text { Dumigan DG, Boyce JM, } \\
\text { Havill NL, Golebiewski M, } \\
\text { Bologun O, Rizvani R. }\end{array}$ & $\begin{array}{l}\text { Am J Infect Control. 38: 387-92, } \\
2010 .\end{array}$ \\
\hline $\begin{array}{l}\text { Condition of cleanliness of } \\
\text { surfaces close to patients in an } \\
\text { intensive care unit. }\end{array}$ & Brasil & $\begin{array}{l}\text { Ferreira AM, Andrade D, } \\
\text { Rigotti MA, Ferreira MVF. }\end{array}$ & $\begin{array}{l}\text { Rev Lat Am } \\
\text { Enfermagem.19(3):557-64, } 2011 .\end{array}$ \\
\hline $\begin{array}{l}\text { Use of audit tools to evaluate the } \\
\text { efficacy of cleaning systems in } \\
\text { hospitals. }\end{array}$ & Reino Unido & $\begin{array}{l}\text { Malik RE, Cooper RA, } \\
\text { Griffith CJ. }\end{array}$ & $\begin{array}{l}\text { Am J Infect Control. } \\
\text { 31: 181-7, } 2003 .\end{array}$ \\
\hline $\begin{array}{l}\text { Finding a benchmark for } \\
\text { monitoring hospital cleanliness. }\end{array}$ & Reino Unido & $\begin{array}{l}\text { Mulvey D, Redding P, } \\
\text { Robertson C, Woodall C, } \\
\text { Kingsmore P, Bedwell D, } \\
\text { Dancer SJ. }\end{array}$ & J Hosp Infect.77(1):25-30, 2011. \\
\hline
\end{tabular}




\begin{tabular}{|c|c|c|c|}
\hline Título do artigo & País & Autores & $\begin{array}{c}\text { Periódicos } \\
\text { (volume, número, página, ano) }\end{array}$ \\
\hline $\begin{array}{l}\text { Validation and comparison of } \\
\text { three adenosine triphosphate } \\
\text { luminometers for monitoring } \\
\text { hospital surface sanitization: } \\
\text { A Rosetta Stone for adenosine } \\
\text { triphosphate testing. }\end{array}$ & Estados Unidos & Sciortino CV, Gilles RA. & $\begin{array}{l}\text { Am J Infect Control. 40:233-9, } \\
2012 .\end{array}$ \\
\hline $\begin{array}{l}\text { The use of adenosine triphosphate } \\
\text { bioluminescence to assess the } \\
\text { efficacy of a modified cleaning } \\
\text { program implemented within an } \\
\text { intensive care setting. }\end{array}$ & Reino Unido & $\begin{array}{l}\text { Moore G, Smyth D, } \\
\text { Singleton J, Wilson P. }\end{array}$ & $\begin{array}{l}\text { Am J Infect Cont. 38:617-22, } \\
2010 .\end{array}$ \\
\hline $\begin{array}{l}\text { Comparison of fluorescent marker } \\
\text { systems with } 2 \text { quantitative } \\
\text { methods of assessing terminal } \\
\text { cleaning practices. }\end{array}$ & Estados Unidos & $\begin{array}{l}\text { Boyce JM, Havill NL, } \\
\text { Havill HL, Mangione E, } \\
\text { Dumigan DG, Moore BA. }\end{array}$ & $\begin{array}{l}\text { Infect Control Hosp } \\
\text { Epidemiol.32(12):1187-93, } 2011 .\end{array}$ \\
\hline $\begin{array}{l}\text { Do surface and cleaning } \\
\text { chemistries interfere with } \\
\text { ATP measurement systems for } \\
\text { monitoring patient room hygiene? }\end{array}$ & Estados Unidos & $\begin{array}{l}\text { Brown E, Eder AR, } \\
\text { Thompson KM. }\end{array}$ & J Hosp Infect. 74:193-5, 2010. \\
\hline $\begin{array}{l}\text { Is it really clean? An evaluation of } \\
\text { the efficacy of four methods for } \\
\text { determining hospital cleanliness. }\end{array}$ & Irlanda & $\begin{array}{l}\text { Sherlock O, O'Connell N, } \\
\text { Creamer E, H Humphreys. }\end{array}$ & J Hosp Infect.72: 140-6, 2009. \\
\hline $\begin{array}{l}\text { The effectiveness of existing and } \\
\text { modified cleaning regimens in a } \\
\text { Welsh hospital. }\end{array}$ & Reino Unido & $\begin{array}{l}\text { Griffith CJ, Obee P, } \\
\text { Cooper RA, Burton NF, } \\
\text { Lewis M. }\end{array}$ & J Hosp Infect.66:352-9, 2007. \\
\hline $\begin{array}{l}\text { A modified ATP benchmark for } \\
\text { evaluating the cleaning of some } \\
\text { hospital environmental surfaces. }\end{array}$ & Reino Unido & $\begin{array}{l}\text { Lewis T, Griffith C, } \\
\text { Gallo M, Weinbren M. }\end{array}$ & J Hosp Infect.69:156-63, 2008. \\
\hline $\begin{array}{l}\text { Estimating bacterial surface } \\
\text { contaminationby means of ATP } \\
\text { determinations: } 20 \text { pence short of } \\
\text { a pound. }\end{array}$ & Reino Unido & Malik DJ, Shama G. & J Hosp Infect.80: 354-6,2012. \\
\hline $\begin{array}{l}\text { Variations in hospital daily } \\
\text { cleaning practices. }\end{array}$ & Estados Unidos & $\begin{array}{l}\text { Boyce JM, Havill NL, } \\
\text { Lipka A, Havill H, Rizvani R. }\end{array}$ & $\begin{array}{l}\text { Infect Control Hosp } \\
\text { Epidemiol.31:99-101, } 2010 .\end{array}$ \\
\hline
\end{tabular}

Para a finalidade de monitorar a efetividade da limpeza de superfícies em estabelecimentos de saúde o ATP bioluminescência foi apontado como importante ferramenta educacional e, principalmente, como método complementar à inspeção visual e aos métodos microbiológicos ${ }^{(11,18,21,24-26)}$.

As principais controvérsias identificadas na literatura quanto ao uso do ATP bioluminescência para avaliar a efetividade da limpeza foram: a discordância entre os resultados do método e aqueles obtidos por análise microbiológica; a possibilidade de interferência na leitura do ATP por substâncias químicas, como detergentes, desinfetantes e plastificantes; a variabilidade nos resultados conforme o instrumento utilizado, além da diversidade de valores de referência propostos para a interpretação dos resultados, segundo a marca e o modelo do instrumento ${ }^{(7,12,18-22,27}$.

A ausência de correlação entre a leitura de ATP e a contagem de colônias foi observada em 53,3\% (8/15) dos estudos analisados. Tal fato foi atribuído à capacidade do ATP bioluminescência de detectar matéria orgânica microbiana e não microbiana em contraste com o método microbiológico que avalia somente a presença de micro-organismos aeróbicos ${ }^{(7,13,18,21,24-27)}$.

O reconhecimento das substâncias químicas como fatores intervenientes na leitura do ATP foi registrado em 20,0\% (3/15) das publicações. Dentre estas se destacaram a albumina sérica bovina 5,0\%, hipoclorito de sódio a 10,0\%, compostos de quaternário de amônio e detergentes ${ }^{(19,22,27)}$.

Plastificantes de panos de microfibra, assim como a composição e a condição de conservação das superfícies analisadas também foram associados às leituras de ATP falso-positivas ${ }^{(22)}$.

O desempenho de três luminômetros de ATP, disponíveis para uso em estabelecimentos de saúde, foram testados e comparados quanto a capacidade de recuperação de ATP microbiano de superfícies. Os resultados variaram conforme o instrumento utilizado e o operador do sistema. Padronização da técnica, treinamento do profissional, múltiplas amostragens 
das superfícies e ajustes no aparelho, tais como calibração, foram medidas indicadas para o controle da interferência decorrente da subjetividade do manipulador ${ }^{(19,22)}$.

O formato do swab foi considerado um importante fator para a recuperação de ATP das superfícies. Swabs ovais e com superfícies planas propiciaram maior contato e melhor adaptação às superfícies analisadas favorecendo maior detecção de ATP. Estes obtiveram $55,3 \% \pm 12,3 \%$ de eficiência na recuperação de Staphylococcus aureus, enquanto os outros $13,3 \% \pm 4,4 \%$ e $7,2 \% \pm 7,2 \%{ }^{(19)}$. Reagentes utilizados no sistema ATP com limitada capacidade para a lise de micro-organismos foram associados ao aumento do limite de detecção da adenosina trifosfato, ou seja, à menor sensibilidade do sistema ${ }^{(19)}$.

A interpretação dos resultados obtidos pelo método de ATP bioluminescência foi reconhecida como uma das maiores dificuldades para a sua aplicação(18).

Nos estudos analisados, a limpeza foi considerada apropriada quando as leituras do ATP registraram valores inferiores a 25 URL, 100 URL, 250 URL ou a 500 URL, de acordo com a marca e o modelo do equipamento utilizado ${ }^{(7,11,13-14,18,20-21,24-26,28)}$.

Quando instituídos valores de referência para o ATP $<100$ URL, aproximadamente $60 \%$ das análises de limpeza das superfícies realizadas utilizando este método coincidiram com aquelas decorrentes da contagem de colônias aeróbicas totais e que indicavam crescimento microbiano inferior a 2,5 $\mathrm{UFC} / \mathrm{cm}^{2(18)}$.

\section{DISCUSSÃO}

O uso do ATP bioluminescência nos serviços de saúde pode configurar-se como importante estratégia educacional, visto que fornece para a equipe evidências imediatas de falhas no processo de limpeza, através da detecção de ATP residual sobre as superfícies, e como método complementar à avaliação visual e microbiológica. Entretanto, a baixa sensibilidade e especificidade do teste, bem como a dificuldade para a interpretação dos resultados tem comprometido a adoção efetiva deste nos serviços de saúde para avaliação da limpeza de superfícies.

Uma vez que o método capta ATP de diversas fontes de matéria orgânica, seja ela microbiana ou não microbiana, tais como excreções, secreções humanas, sangue e restos de alimentos, os resultados obtidos não correspondem, necessariamente, à contaminação do ambiente por micro-organismos e, consequentemente, não pode ser relacionado ao risco do paciente adquirir patógenos. A impossibilidade de correlação entre os níveis de ATP e de contaminação microbiana de uma superfície constitui um dos principais empecilhos para o uso

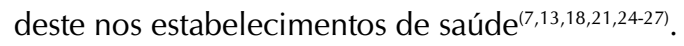

As diferenças observadas nos estudos analisados entre o percentual de superfícies identificadas como contaminadas pelo método de ATP e pela contagem de colônias aeróbicas totais $(80,0 \% \times 16,0 \% ; 55,0 \% \times 23 \% ; 28,5 \% \times 7,9 \%)$ condizem com achados da literatura de que, em média, 33,0\% do ATP presente nas superfícies frequentemente tocadas no ambiente hospitalar são de origem microbiana $a^{(11,13,21,24,26)}$.
Uma alternativa para a distinção do ATP de origem microbiana dos demais seria a remoção enzimática do ATP não microbiano da superfície, previamente a aplicação do teste. No entanto, a viabilidade e a identificação dos micro-organismos recuperados, ainda assim permaneceriam desconhecidas ${ }^{(16)}$.

Para haver uma forte correlação entre os níveis de ATP e a presença de organismos viáveis, a proporção entre as fontes de ATP (microbiana e não microbiana) nas superfícies deveriam ser constantes, e há diversas razões para esta não ocorrer. Uma delas refere-se ao fato de que os níveis de ATP intracelular não são idênticos para todos os micro-organismos. Além disso, alguns micro-organismos são aparentemente capazes de regular os níveis de ATP em resposta a estressores ambientais. Há indícios que Mycobacterium tuberculosis, quando carente de nutrientes, seja capaz de reduzir os níveis de ATP a $1 / 5$ da concentração basal ${ }^{(26-27)}$.

Embora os resultados fornecidos pelo sistema de ATP bioluminescência possam ser comprometidos principalmente pela inespecificidade do método em captar a energia de diversas matérias orgânicas e não somente aquelas derivadas de micro-organismos, alguns estudos têm identificado que fatores ambientais também podem influenciar as leituras do sistema ${ }^{(12,18,22,27)}$.

Substâncias químicas comumente utilizadas no processo de limpeza ou desinfecção das superfícies hospitalares como os detergentes, compostos de amônio e hipoclorito de sódio podem interferir nas leituras de ATP comprometendo a confiabilidade dos resultados. Trata-se de inquestionável limitação do método, principalmente em situações de surto de Clostridium difficile e Norovírus, nas quais o uso do hipoclorito de sódio a $10 \%$ é mandatório ${ }^{(8,12,18-9,22,27)}$.

Por estas razões, o sistema de ATP bioluminescência tem sido associado à sensibilidade e especificidade demasiadamente baixa ( $57 \%$ ) na detecção de bactérias e a sua utilização indicada para programas educacionais e avaliações do processo de limpeza preferencialmente associado aos métodos microbiológicos ${ }^{(16,18,22)}$.

O uso complementar de diferentes métodos para avaliação da efetividade da limpeza de superfícies, visto que utilizam parâmetros distintos, pode contribuir para o gerenciamento da limpeza hospitalar e favorecer a relação entre contaminação de superfícies e taxas de infecção. Contudo, os custos e o tempo envolvidos podem inviabilizar a adoção desta prática ${ }^{(18,20,25)}$.

Uma vez definido como método de escolha para determinar a eficácia da limpeza, o sistema de ATP bioluminescência deve ser cuidadosamente analisado e validado. Para tanto, este deve ser testado em cada unidade hospitalar, a fim de estimar a sua aplicabilidade mediante os níveis de contaminação e limpeza rotineiramente presentes naquele ambiente, e os resultados obtidos devem ser analisados considerando as características do setor e as especificidades do aparelho utilizado, tal como o valor de referência indicado ${ }^{(20)}$.

Similar a outros métodos, não está claro que os valores de referência sugeridos para determinar a efetividade da limpeza de uma superfície pelo ATP bioluminescência sejam adequados. Valores de 25 a 500 URL têm sido utilizados como referência, conforme a sensibilidade do luminômetro e as características da unidade investigada ${ }^{(29)}$. 
Concentrações de ATP inferiores a 500 URL tem sido propostas para designar superfícies limpas. Acredita-se que quando empregadas as melhores práticas de limpeza, esta se torna uma referência objetiva e atingível. Por outro lado, resultados abaixo de 25 URL tem sido considerados demasiadamente rigorosos com elevado risco de reprovação do processo de limpeza e associado ao aumento dos custos e do mau emprego do tempo dos profissionais envolvidos ${ }^{(18,25-26,29)}$.

Nas Unidades de terapia intensiva, nas quais a contaminação ambiental é particularmente importante assim como o risco de colonização e infecção do paciente por micro-organismos diversos, a meta de 250 URL tem sido considerada $a^{(1,6,26,30)}$.

O limite de 100 URL tem sido apontado como aquele que mais se aproxima dos níveis de crescimento microbiano inferiores a 2,5 UFC/ $/ \mathrm{cm}^{2}$, porém determiná-lo como padrão de referência implicaria no uso de um único aparelho de ATP bioluminescência, visto que nem todos os modelos apresentam a mesma sensibilidade ${ }^{(18,31)}$.

Estudos que avaliaram superfícies pelo método de ATP observaram que algumas destas, comumente, apresentavam leituras de ATP inferiores ao valor de referência recomendado antes mesmo da execução da limpeza. Esses achados reiteram a necessidade de se conhecer os níveis de limpeza rotineiramente alcançados para o estabelecimento de valores de referência adequados à realidade local e a obtenção de resultados confiáveis capazes de expressar a qualidade e eficácia do processo de limpeza ${ }^{(20,25-26)}$.

Apesar das controvérsias para o uso do ATP bioluminescência na avaliação da efetividade da limpeza de superfícies, este tem sido considerado como importante ferramenta educacional, uma vez que permite identificar o nível de limpeza alcançado, o cumprimento das práticas recomendadas, bem como a divulgação imediata dos resultados à equipe envolvida nos processos de limpeza. Se comparado a outros testes, apresenta, ainda, como vantagem, a obtenção de resultados rápidos e quantitativos, associados à técnica de fácil aplicação. Entretanto, por se tratar de método ainda pouco aplicado nos serviços de saúde, e cuja abordagem do tema é, ainda, escassa, mais estudos se fazem necessários para a definição dos melhores padrões a serem seguidos e da repercussão dos seus resultados para a segurança do paciente ${ }^{(12,21,24)}$.

\section{CONCLUSÃO}

O ATP bioluminescência tem sido indicado como recurso educacional e método complementar às análises microbiológicas, porém sua aplicação nos estabelecimentos de saúde é, ain$\mathrm{da}$, recente e tem se configurado em alvo de diversas discussões.

A pequena correlação entre as leituras de ATP e a contaminação de uma superfície por micro-organismos viáveis, a dificuldade de interpretação e comparação dos resultados, visto a variedade de valores de referência propostos e as peculiaridades de cada sistema disponível para uso, bem como a possibilidade de interferência por substâncias químicas, constituem as principais controvérsias para o uso do método nos estabelecimentos de saúde, sobre as quais deverão basear-se mais estudos a fim de estabelecer a real contribuição do ATP bioluminescência para a avaliação da limpeza de superfícies.

\section{REFERÊNCIAS}

1. Siegel JD, Rhinehart E, Jackson M, Chiarello L; Healthcare Infection Control Practices Advisory Committee. Guideline for isolation precautions: preventing transmission of infectious agents in healthcare settings 2007. Atlanta (GA): Centers for Disease Control and Prevention; 2007.

2. Datta R, Platt R, Yokoe DS, Huang SS. Environmental cleaning intervention and risk of acquiring multidrug-resistant organisms from prior room occupants. Arch Inter Med [Internet].2011 [cited 2013 May 06];171(6):491-4. Available from: http://www.ncbi.nlm.nih.gov/pubmed/21444840

3. Oliveira AC, Damasceno QS, Piscoya M, Nicoli JR. Epidemiologic characteristics of resistant microorganisms present in reserves from an intensive care. Am J Infect Control [Internet]. 2012 [cited 2013 May 06];40(2):1-3.

4. Dancer SJ. The role of environmental cleaning in the control of hospital-acquired infection. J Hosp Infect [Internet]. 2009 [cited 2013 May 06];73(4):378-85. Available from: http://www.ncbi.nlm.nih.gov/pubmed/19726106

5. Sexton T, Clark P, O'neill E, Dillane T, Humphreys $H$. Environmental reservoirs of methicillin-resistant Staphylococcus aureus in isolation rooms: correlation with patient isolates and implications for hospital hygiene. J Hosp Infect [Internet]. 2006 [cited 2013 May
06];62(2):187-94. Available from: http://www.ncbi.nlm. nih.gov/pubmed/16290319

6. Huang SS, Datta R, Platt, R. Risk of acquiring antibiotic-resistant bacteria from prior room occupants. Arch Inter Med [Internet]. 2006 [cited 2013 May 06];166(18):1945-51. Available from: http://www.ncbi.nlm.nih.gov/pubmed/17030826

7. Boyce JM, Havill NL, Dumigan DG, Golebiewski M, Balogun O, Rizvani R. Monitoring the effectiveness of hospital cleaning practices by use ofan adenosine triphosphate bioluminescence assay. Infect Control Hosp Epidemiol [Internet]. 2009 [cited 2013 May 06];30(7):678-84. Available from: http://www.ncbi.nlm.nih.gov/pubmed/19489715

8. Agência Nacional de Vigilância Sanitária (BR). Segurança do paciente em serviços de saúde: limpeza e desinfecção de superfícies [Internet]. Brasília (DF): Anvisa; 2010 [acesso em 06 de maio de 2013]. Disponível em: http://portal. anvisa.gov.br/wps/wcm/connect/4ec6a200474592fa9b3 2df3fbc4c6735/Manual + Limpeza +e + Desinfeccao + W EB.pdf?MOD = AJPERES

9. Healthcare infection control practices advisory committee (HICPAC). Guideline for disinfection and sterilization in healthcare facilities [Internet]. Atlanta: HIPAC; 2008 [cited 2013 May 06]. Available from: http://www.cdc.gov/ 
hicpac/pdf/guidelines/disinfection_nov_2008.pdf

10. Dancer SJ. How do we assess hospital cleaning? A proposal for microbiological standards for surface hygiene in hospitals. J Hosp Infect [Internet]. 2004 [cited 2013 May 06];56(1):10-5. Available from: http://www.ncbi. nlm.nih.gov/pubmed/14706265

11. Griffith CJ, Cooper RA, Gilmore J, Davies C, Lewis M. An evaluation of hospital cleaning regimes and standards. J Hosp Infect [Internet]. 2000 [cited 2013 May 06];45(1):19-28. Available from: http://www.ncbi.nlm. nih.gov/pubmed/10833340

12. Dumigan DG, Boyce JM, Havill NL, Golebiewski M, Bologun $\mathrm{O}$, Rizvani $\mathrm{R}$. Who is really caring for your environment of care? Developing standardized cleaning procedures and effective monitoring techniques. Am J Infect Control [Internet]. 2010 [cited 2013 May 06];38(5):387-92. Available from: http://www.ncbi.nlm. nih.gov/pubmed/20435376

13. Ferreira AM, Andrade D, Rigotti MA, Ferreira MVF. Condition of cleanliness of surfaces close to patients in an intensive care unit. Rev Latinoam Enferm. 2011;19(3):557-64.

14. Malik RE, Cooper RA, Griffith CJ. Use of audit tools to evaluate the efficacy of cleaning systems in hospitals. Am J Infect Control [Internet]. 2003 [cited 2013 May 06];31(1):181-7. Available from: http://www.ncbi.nlm. nih.gov/pubmed/12734526

15. Carling PC, Von Beheren S, Kim P, Woods C; Healthcare Environmental Hygiene Study Group. Intensive care unit environmental cleaning: an evaluation in sixteen hospitals using a novel assessment tool. J Hosp Infect [Internet]. 2008 [cited 2013 May 06];68(1):39-44.Available from: http://www.ncbi.nlm.nih.gov/pubmed/18069083

16. Tasmanian infection prevention and control unit. Evaluating environmental cleanliness in hospitals and other healthcare settings. What are the most effective and efficient methods to use? Tasmania: TIPCU; 2012.

17. Guh A, Carling P; Environmental Evaluation Workgroup. Options for Evaluating Environmental [Internet]. [place unknown: publisher unknown]. 2010 [cited 2013 May 06]. Available from: http://www.cdc.gov/HAl/pdfs/toolkits/Environ-Cleaning-Eval-Toolkit12-2-2010.pdf

18. Mulvey D, Redding P, Robertson C, Woodall C, Kingsmore P, Bedwell D, Dancer SJ. Finding a benchmark for monitoring hospital cleanliness. J Hosp Infect [Internet]. 2011 [cited 2013 May 06];77(1):25-30. Available from: http://www.ncbi.nlm.nih.gov/pubmed/21129820

19. Sciortino CV, Gilles RA. Validation and comparison of three adenosine triphosphate luminometers for monitoring hospital surface sanitization: a Rosetta Stone for adenosine triphosphate testing. Am J Infect Control [Internet]. 2012 [cited 2013 May 06];40(8):233-9. Available from: http://www.ncbi.nlm.nih.gov/pubmed/23021416

20. Moore G, Smyth D, Singleton J, Wilson P. The use of adenosine triphosphate bioluminescence to assess the efficacy of a modified cleaning program implemented within an intensive care setting. Am J Infect Control [Internet]. 2010 [cited 2013 May 06];38(8):617-22. Available from: http://www.ncbi.nlm.nih.gov/pubmed/20605265

21. Boyce JM, Havill NL, Havill HL, Mangione E, Dumigan DG, Moore BA. Comparison of fluorescent marker systems with 2 quantitative methods of assessing terminal cleaning practices. Infect Control Hosp Epidemiol [Internet]. 2011 [cited 2013 May 06];32(12):1187-93. Available from: http://www.ncbi.nlm.nih.gov/pubmed/22080657

22. Brown E, Eder AR, Thompson KM. Do surface and cleaning chemistries interfere with ATP measurement systems for monitoring patient room hygiene? J Hosp Infect [Internet]. 2010 [cited 2013 May 06];74(2):193-5. Available from: http://www.ncbi.nlm.nih.gov/pubmed/20060618

23. Mendes KDS, Silveira RCCP, Galvão CM. Revisão integrativa: método de pesquisa para a incorporação de evidências na saúde e na enfermagem. Texto \&Contexto Enferm [Internet]. 2008 [acesso em 06 de maio de 2013];17(4):758-64. Disponível em: http:// www.scielo.br/scielo.php?script $=$ sci arttext\&pid $=$ S0104-07072008000400018

24. Sherlock O, O'Connell N, Creamer E, Humphreys H. Is it really clean? An evaluation of the efficacy of four methods for determining hospital cleanliness. J Hosp Infect [Internet]. 2009 [cited 2013 May 06];72(2):140-6. Available from: http://www.ncbi.nlm.nih.gov/pubmed/19321226

25. Griffith CJ, Obee P, Cooper RA, Burton NF, Lewis M. The effectiveness of existing and modified cleaning regimens in a Welsh hospital. J Hosp Infect [Internet]. 2007 [cited 2013 May 06];66(4):352-9. Available from: http://www. ncbi.nlm.nih.gov/pubmed/17655976

26. Lewis T, Griffith C, Gallo M, Weinbren M. A modified ATP benchmark for evaluating the cleaning of some hospital environmental surfaces. J Hosp Infect [Internet]. 2008 [cited 2013 May 06];69(2):156-63. Available from: http://www.ncbi.nlm.nih.gov/pubmed/18468725

27. Malik DJ, Shama G. Estimating bacterial surface contamination by means of ATP determinations: 20 pence short of a pound. J Hosp Infect. 2012;80(4):354-6.

28. Boyce JM, Havill NL, Lipka A, Havill H, Rizvani R. Variations in hospital daily cleaning practices. Infect Control Hosp Epidemiol [Internet]. 2010 [cited 2013 May 06];31(1):99-101. Available from: http://www.ncbi.nlm. nih.gov/pubmed/19951203

29. Dancer S. Hospital cleaning in the 21 st century. Eur J Clin Microbiol Infect Dis [Internet]. 2011 [cited 2013 May 06];30(12):1473-81. Available from: http://www.ncbi. nlm.nih.gov/pubmed/21499954

30. Siegel JD, Rhinehart E, Jackson M, Chiarello L; Healthcare Infection Control Practices Advisory Committee (HICPAC). Management of multidrug-resistant organisms in healthcare settings [Internet]. 2006 [cited 2013 May 06]. Atlanta (US): Centers for Disease Control and Prevention; 2006. Available from: http://www.cdc.gov/hicpac/pdf/ guidelines/MDROGuideline2006.pdf

31. Dancer SJ. Importance of the environment in meticillin-resistant Staphylococcus aureus acquisition: the case for hospital cleaning. Lancet Infect Dis [Internet]. 2008 [cited 2013 May 06];8(2):101-13. Available from: http://www. ncbi.nlm.nih.gov/pubmed/17974481 